\title{
Crystalline Metals Effortlessly Fit the Mold
}

\author{
Molding crystalline metals like silver into nanopillar structures is both possible and easier to \\ achieve for narrower pillars, in contrast with other materials.
}

\section{by Suveen N. Mathaudhu*,†}

$\mathrm{I}$ $\mathrm{n}$ the pockets of much of the world's population sit molded crystalline metals decorated with micrometerscale features-coins. Coins have circulated for more than 2.5 millennia [1], taking on many forms and having varied decorations. The patterns on the first gold and silver coins were likely made by heating the metals and then pressing them between two imprint molds (Fig. 1). But despite this long history, researchers have refrained from molding smaller, nanometer-sized features in crystalline metals, assuming that it would be impossible. Now, Ze Liu from Wuhan University in China and colleagues show that nanomolding of high-aspect-ratio features in silver is possible. They also show that molding becomes easier as the width of the feature decreases-not harder, as with other material types [2]. This finding boosts the number of

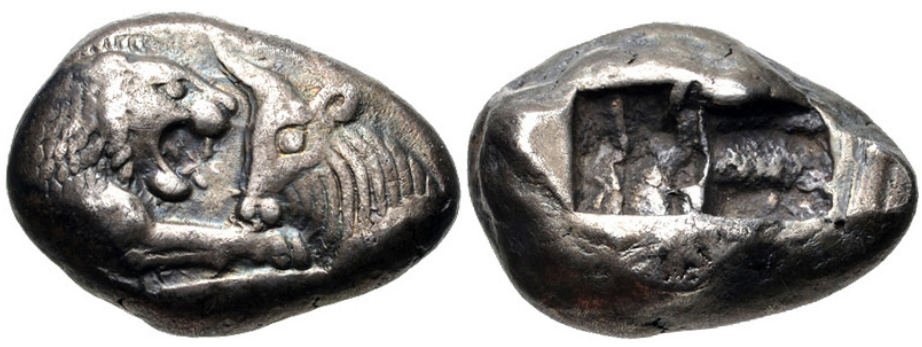

Figure 1: King Croesus of Lydia (now part of Turkey) introduced the first gold and silver coins into circulation in around 550 BCE (Before the Common Era). The image shows a silver coin created from this period. The coin is approximately $5 \mathrm{~mm}$ in diameter. The left-hand image shows the front of the coin, which is imprinted with the heads of a lion and a bull. The right-hand image shows the back of the coin, with an impression from the back of the mold. (CNG Coins/Wikimedia Commons)

* Department of Mechanical Engineering and Materials Science and Engineering Program, University of California, Riverside, CA, USA $\dagger$ Energy and Environment Directorate, Pacific Northwest National Laboratory, Richland, WA, USA methods for creating nanostructures-used in technologies ranging from food and water purification to the sensing of biological molecules-from crystalline metals.

For decades, researchers have strived to structure silver on the nanoscale. The mechanical, optical, electronic, and catalytic properties of the resulting patterns are utilized in myriad technologies related to health, energy, and defense [3]. For example, nanosized pillars can display antibacterial properties [4]. Robust methods for molding nanopillars exist for materials that soften as they approach their melting temperature, such as polymers, gels, and glasses. However, nanomolding of silver and other crystalline metals has proven elusive [5]. Instead, researchers typically pattern silver using lithography methods, in which the pattern is etched into the material. But lithography methods are time consuming and costly and cannot produce high-aspect-ratio structures.

One factor thought to influence molding of tiny structures in polymers, gels, and glasses is their small flow unit-the individual unit in the material that flows. For a sand timer the flow unit is a sand grain; for a polymer it's a molecule. The flow unit of a polymer can be as small as $1 \mathrm{~nm}$, making nanomold filling relatively easy. That said, creating high-aspect-ratio features, such as tall nanopillars, using polymers is still tricky. For example, a large pressure has to be applied to the material for it to overcome the high capillary forces associated with narrow features and to fill the mold. Because of this, creating such structures from polymers becomes harder as the diameters of the pillars decrease. For molten silver and other crystalline metals, the flow unit is the size of an individual atom. However, the high viscosity and high capillary forces of liquid silver stop it from deeply filling the mold and results in short, stubby pillars. Nanomolding is typically performed at temperatures well below silver's melting point, where the metal is crystalline, albeit slightly softened. In such a scenario, silver does not flow like a liquid. The material can still deform via other mechanisms, but none are thought to be relevant for nanomolding.

Liu and colleagues now show a way to mold crystalline metals into nanostructures, and they find that-unlike polymers-molding metals into tall, thin pillars is easier for 


\section{Phys̄īcs}

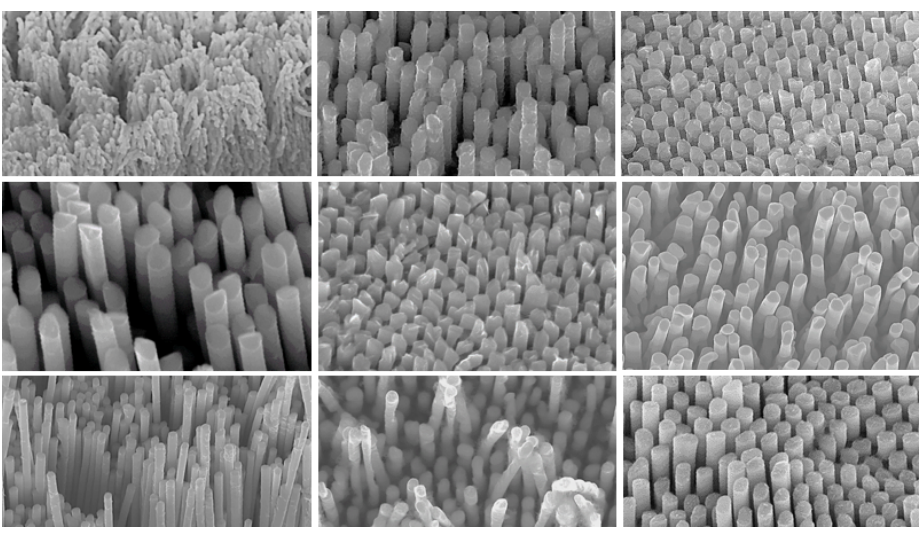

Figure 2: Electron micrographs showing examples of the nanopillars created by Liu and colleagues from silver, nickel, vanadium, iron, and various metal alloys. The silver pillars (top left corner) have diameters between 5 and $13 \mathrm{~nm}$ [2]. (Z. Liu et al. [2])

narrower pillars. To create pillar-shaped structures, the team used molds containing an array of pits with decreasing diameters. The pits ranged in diameter from 5 to $320 \mathrm{~nm}$. They placed a disk of pure silver on top of the mold and pressed down on the disk with a heated plate, pushing the metal into the holes. The pressure, temperature, and molding time were kept constant for each experiment. After cooling the metal and removing the pattern from the mold, the team measured the length of the resulting pillars.

The team found that the length-to-diameter ratio of the nanopillars increased as the diameters of the pits decreased, a finding that indicates that it's easier to mold smaller features in this material. Remarkably, the team was able to produce 5-nm-wide silver pillars with heights of around $1700 \mathrm{~nm}$, making them among the highest-aspect-ratio pillars ever achieved for metallic materials.

The question then arises of how this counterintuitive feat is possible. At molding temperatures, crystalline metals typically deform via one or more of the following mechanisms: the propagation of defects through the material, which causes crystal planes to glide past one another; the shift or rotation of crystals relative to one another, which results in the material flowing; and the coordinated displacement of atoms along a crystal plane [6]. The team ruled out all of these possibilities in their experiments. Instead, their observations point to another mechanism, that of diffusion.

The silver disks used by the team were imperfect-they contained defects, known as vacancies, where atoms are missing in the lattice. Atoms can diffuse through the disk by jumping into a vacancy site. Changing the shape of a crystalline material via diffusion requires the continuous movement of atoms along internal vacancy pathways or along the interface between the material and the mold wall. It is known that the amount of internal diffusion-known as lattice diffusion-scales as $1 / d$, where $d$ is the diameter of the pillar. For molding of large structures, this scaling means that lattice diffusion has little effect because the abovementioned conventional deformation mechanisms are energetically favorable. However, the team predicts that enhanced diffusion is possible for nanosized structures made with their technique, facilitating the flow of silver into the nanopits.

The team's theory and experimental observations explain how such enhanced diffusion can take place. Pushing down on the silver disk creates a pressure gradient in the material, with the lowest pressure occurring just before the entrance to a pit and the pressure increasing just after it. As a result, the silver in the pits contains more vacancies, and lattice diffusion in this region is faster. As the pits get smaller, the pressure jump increases, and so does diffusion, explaining why the narrow pillars grow faster. For silver, when the pits are narrower than $10 \mathrm{~nm}$, the data suggest that diffusion becomes even more efficient, with the amount of diffusion scaling as $1 / d^{2}$.

The behavior observed by Liu and colleagues for the molding of silver nanopillars should apply to all crystalline metals, as atomic diffusion is omnipresent. Indeed, they obtained initial results that replicate their data for silver, pure iron, vanadium, and nickel, as well as for a variety of metal alloys (Fig. 2).

The ability to mold high-aspect-ratio nanometer-width pillars from crystalline metals provides a pathway for a deeper understanding of how these materials deform on this length scale. It also opens the door to creating nanowires and studying their properties. From a broader technological perspective, this quick and cheap method for forming nanostructures could spur researchers to uncover new applications for crystalline-metal-based nanomaterials in as-yetunknown technologies.

This research is published in Physical Review Letters.

\section{REFERENCES}

[1] N. Cahill and J. H. Kroll, "New archaic coin finds at Sardis," Am. J. Archaeol. 109, 589 (2005).

[2] Z. Liu, G. Han, S. Sohn, N. Liu, and J. Schoers, "Nanomolding of crystalline metals: The smaller the easier," Phys. Rev. Lett. 122, 036101 (2019).

[3] F. Matteucci, R. Giannantonio, F. Calabi, A. Agostiano, G. Gigli, and M. Rossi, "Deployment and exploitation of nanotechnology nanomaterials and nanomedicine," AIP Conf. Proc. 1990, 02001 (2018).

[4] S. Pogodin et al., "Biophysical model of bacterial cell interactions with nanopatterned cicada wing surfaces," Biophys. J. 104, 835 (2013).

[5] Z. Liu, "One-step fabrication of crystalline metal nanostructures by direct nanoimprinting below melting temperatures," Nat. Commun. 8, 14910 (2017). 


\section{Phys̄īcs}

[6] H. J. Frost and M. F. Ashby, Deformation mechanism maps: The plasticity and creep of metals and ceramics (Pergamon Press,

Oxford, 1982). 